REVIEW

\title{
Nursing and Rehabilitative Care of the Elderly Using Humanoid Robots
}

\author{
Tetsuya Tanioka \\ Institute of Biomedical Sciences, Tokushima University, Graduate School, Tokushima, Japan
}

\begin{abstract}
Japan's declining birth rate and increasing aging population prompted intercessory efforts towards robot technologies in nursing practice for the elderly. Today, technological companies are developing robots that meet universal health care technology demands. While human caring focus on human-to-human relationships, but between humans and nonhumans, e.g. Humanoid Nursing Robot (HNRs)-to-human relationships, caring practices have not been forthcoming. When HNRs can support patients independently, capabilities much like being human will be required, including intelligence and skill competencies. Currently, Tanioka's research group is conducting clinical trials of humanoid robots equipped with applications using Pepper (manufactured by SOFTBANK CORPORATION), towards elderly care and rehabilitation at the Mifune Hospital, Kagawa prefecture. Care Prevention Gymnastics Exercises (Pepper-CPGE) was madeby Xing Company, Japan. Therefore, this paper aims to describe the clinical trial outcomes based on the Transactive Relationship Theory of Nursing (TRETON) (Tanioka, 2017) emphasizing nursing engagement processes between HNRs and human persons. Observable effects include positive changes in relationships of patients, humanoid robots and healthcare providers. Emphasizing ethical concerns and human person safety as critical factors of care, and fears for divergent robot use are observed. Cooperative undertakings with various interdisciplinary activities mark the visioning of Japanese human caring ideas for an aging society. J. Med. Invest. 66 :19-23, February, 2019
\end{abstract}

Keywords : Nursing, Rehabilitative Care, Humanoid Nursing Robots (HNRs), Transactive relationship, the Elderly

\section{INTRODUCTION}

Japan has the highest proportion of older adults in the world. Aging is not only an immediate personal issue but also a salient factor in crucial public policies, such as in pensions, health care, and long-term care (1). With its aging population and declining birth rate, Japan will arguably face a shortage of care workers in the coming years (2). High technology robots for use not only in factories but also for our living environment has been developed. Particularly, and rapidly spreading are the use of human-interactive robots for psychological enrichment providing services by interacting with humans while stimulating their minds $(3,4)$.

In response to these identified needs and demands, it is now a reality that technical capabilities possessed by healthcare robots are dramatically evolving. Tanioka's team's research studies $(4,5,6$, 7) were conducted focused on topics such as "what is a healthcare robot (HR), humanoid nursing robot (HNR) or humanoid caring robot (HCR)?" "What nursing tasks physically performed by human nurses can be performed efficiently by HNRs?" "What are the possibilities that HNRs play in stereotypical human-nurse behaviors?"

Nevertheless, there is a dearth of nursing literature addressing how nurses will assist these vulnerable populations in the selection and use of robotic technology or how robotics could affect nursing care and patient outcomes (8). Technological companies are developing various robots to meet universal health care technological demands. There has been changes in the relationship among patients, robots and healthcare providers (9). This relationship

Received for publication November 1, 2018 ; accepted November 1, 2018. Address correspondence and reprint requests to Tetsuya Tanioka, RN ; PhD, FAAN, 3-18-15, Kuramoto-cho, Tokushima 770-8509, Japan and Fax : +81-88-633-9021. advances the link that emphasizes ethical concerns and human person safety as main factors $(10,11)$. Current discourse include concerns and fears of diverse robot use, e.g. issues such as whether or not to save lives, create life, and extend human life.

While human caring focus on human-to-human relationships, in a nonhuman-to-human relationship such as in the case of HNRs, it is essential to be conscious about ethical concerns and human person safety. The increasing population of older persons world-wide fosters the recognition and increasing realization of the need of a theory-based practice model that can guide nursing practice particularly in situations in which HNRs are engaged with human persons as patients. Tanioka's research group is conducting clinical trials using humanoid robots equipped with applications. Pepper (manufactured by SOFTBANK CORP.) with the Care Prevention Gymnastics Exercises (Pepper-CPGE) was made by Xing Company, Japan, is used for elderly care and rehabilitation. The aim of this paper is to describe and explain the clinical trial outcomes using the Transactive Relationship Theory of Nursing (TRETON) for nursing and rehabilitative care engagements between HNRs and human persons.

\section{Transactive Relationship Theory of Nursing (TRETON)}

As the demands and needs for quality health care rise particularly in environments poor in human resources, and among the older adult population, the use of human-machine processes are intensified. The realities and consequences of transactive relationships have become integral assurances for quality human health care.

The Transactive Relationship Theory of Nursing (TRETON) $(12,13)$ explains these engagement processes which are characteristic activities and influential among healthcare providers, humanoid robot, and older adults with dementia. In these situations, the nurse acts as mediator between the older persons and 
the humanoid robot.

The TRETON explains the mutual engagement and technological engagement that transpires between the robot and the human person in the Figure 1 (a). Nursing encounters among HNRs, patient and human nurse in transactive interactions are described within the metaparadigms of person, nursing, environment, and health (14). The central feature of this TRETON is the evolution of sophisticated technologies of care as intelligent machines which are designed to provide nursing care activities for older persons in the light of their scarcity of manpower. The transactions between human beings and healthcare robots are perceived as means of providing and assuring quality healthcare.

Figure 1(b) shows the development process of HNRs. In 2016, the author argued that the possibilities of humanoid nurse robots in healthcare can be possible (9). However, it is important to remember that high-fidelity humanoid robots that can function much like a human being have not yet been developed today. Iwasa (15), a student member of the research team ranked the Pepper as a humanoid robot at level " 0 " based on the proposed behavioral criterion of robots. This indicates that HNRs are still in the developmental stage, and cannot be used as a functional autonomous work robot.

When developing future communication robots for the elderly with dementia, it is critical that an effective conversation program will be designed and developed when this communication robot development will include characteristic transactive relations with nurses as intermediaries.

The role functions of these intermediaries were illustrated to be of high versatility, indicating that situations such as these may also be controlled by other health care providers such as by occupational therapists in their respective practices. Therefore, intermediaries such as healthcare providers are used in a critical role now (16).

\section{The Nursing Encounter : Where All Nursing Occurs}

Nursing persons (nurses and nursing aides) interact with patients within a health care environment. Often, this is within the walls of an institution (hospital or nursing home). The quality of this environment influences the nursing person and the patient. And, the actions of the patient and nursing person are influenced by the attitudes and actions of each other. The caring encounter is an encounter between two equal persons where one is nurse and the other is patient (17). They encounter in mutuality, in true presence, and both have allowed themselves to be the person they are (18). Knowing more about the attributes associated with meaningful encounters makes it possible for caregivers to individualize care for patients and makes it easier to help and support patients in what they most need support with (19).

The coexistence of technology and caring is best exemplified in nursing (20). In Locsin's theory, the attempt was made to delineate the composition, implication, and limitation of technology within the context of human caring (21), in the book, Technological Competency as Caring in Nursing:A Model for Practice. Locsin declared that technology is here to say and that technology, caring, and nursing have become inseparable - coexisting as conceptual models of humanizing care.

The occurrence of a nursing relationships exist between and among human-to-human and human-to-intelligent machines. This kind of nursing encounter (12) is the focal point of engagement between the nurse and nursed. Two distinctive features of this engagement are the human to-human relationship (between the human nurse and human patient), and the technological engagement between the humanoid robot-the intelligent machineand the patient in "its" care. As technological engagements occur, the nursing encounter is illuminated as the shared experience fostering human caring. In order to acknowledge the value of understanding these encounters, the necessary element of "knowing" the patient is initiated.

With robot introduction in hospitals, nursing care facilities and companies are positioning themselves to use various types of robots. However, with a limited understanding of intelligent machine technologies, professional nurses and engineers (10) cannot muster robot functionalities based on the types of robots (22). An ethical analysis of care robot activities suggested that "democratic spaces," should be employed in which all stakeholders in aged care, have a voice in the ethical debate (23). It would involve, at least, a conscious experience when robots, which do not appear to be able to give true caring in the foreseeable future, might be sufficient for taking part in caring, especially when their movements are as if they exhibit care, that is, give the illusion that they respond to the feelings and the suffering of their care recipients (24).

Today, in modern Japan, the humanoid robot Pepper is used as a receptionist for Sushi restaurants (25). It shows cute robot gestures

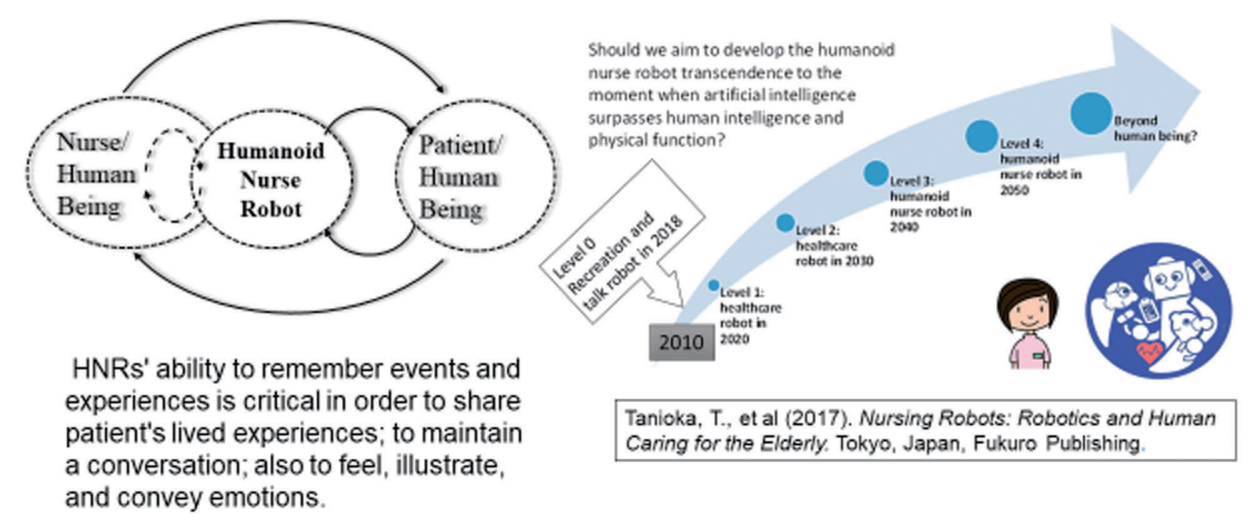

(a) The relationships among nurses, humanoid

(b) Healthcare robots (HRs), humanoid nursing robots nursing robots, and patients. (HNRs), and its future development.

Figure 1. Healthcare robots (HRs), Humanoid nursing robots (HNRs), and its future developme 
by their arms head and eyes. This is an example of a place wherein the nursing encounter occurs with humanoid robots. It is now being used and available in rehabilitation facilities for the elderly. Humanoid robots are becoming mainstay machines as a rehabilitation instructor, and companion of the elderly client.

\section{Clinical trials using humanoid robot}

Tanioka's research group is conducting clinical trials with Pepper robot equipped with applications and used for elderly care and rehabilitation. Figure 2 (a) shows "transactive relationship" among the elderly, the occupational therapist as mediator, and with Pepper robot.

While human caring is focused on human-to-human relationships, essential consideration is the design of other ways of practice. There have been changes in the relationship among patients, robots and healthcare providers work as evidenced in the clinical trial. Emphases on ethical concerns and human person safety are main the factors including concerns and fears of diverse robot use (Figure $2(b))$.
Larger Randomized Controlled Clinical Trial using valid comparators are needed to definitively show where socially assistive robot technology is and is not useful in elderly care (26). The combined results of behavioral observation, actigraphy and heart rate variability (HRV) analyses (27), suggested that persons with dementia showed positive correlations between activity level of the sympathetic nerve (LF/HF) and activity count of actigraph in a wakeful state. Compared to those who showed no positive correlation, they did not require much nursing care.

Figure 3 shows that the sympathetic nervous activity is activated during the ending time of the exercise as compared with the time of the start of the rehabilitation activity and the recreational activity. It is suggested that Humanoid robot's instruction is able to activate the autonomic nervous activity.

This suggests that the Humanoid robot might be able to stimulate improvement of the quality of life of elderly people.

Figure 4 shows the exercise routine conducted with Pepper robot. Tanioka's research group is conducting clinical trials of humanoid robots equipped with applications using the Pepper-
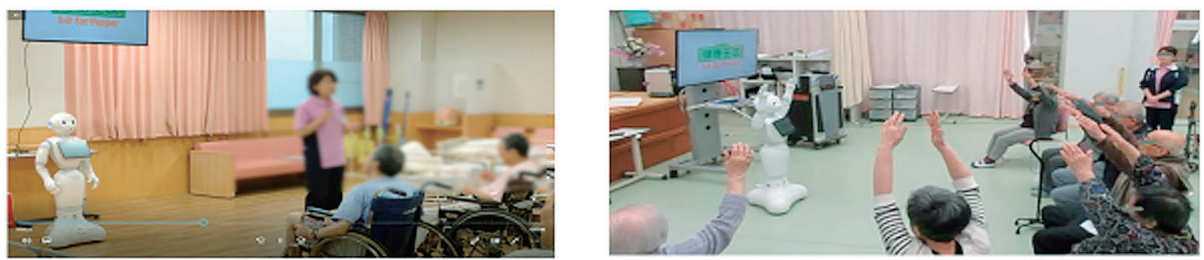

(a) Transactive relationship among elderly, occupational therapist as mediator, and Pepper robot.
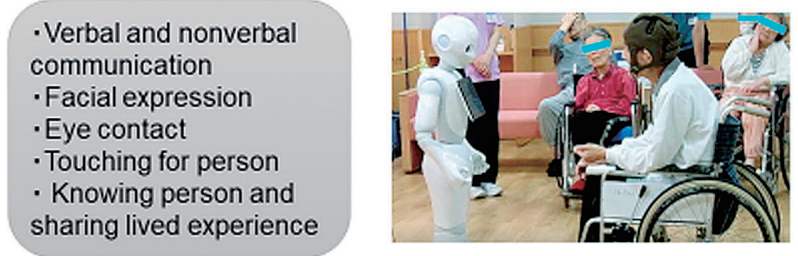

HNRs should be able to correspond according to the mood, feeling, and emotion, including expressions of suffering.

Nurses and healthcare providers are required to make good use of robots thereby understanding patients more fully. Elderly persons can gain well-being from dialogues and conversations with Pepper robots.

(b) Technology and caring coexisting in nursing and healthcare.

Figure2. The Transactive Relationship Theory of Nursing (TRETON) explains what transpires between the robot and the human being

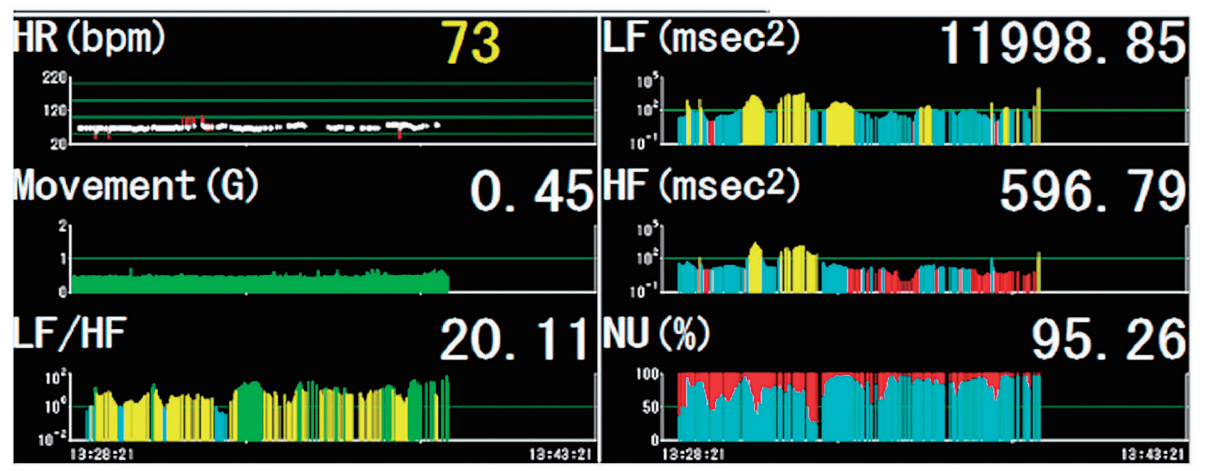

Heart rate variability (HRV) was measured noninvasively to evaluate the sympathetic-parasympathetic balance (MemCalc/Bonaly Light; Suwa Trust, Tokyo, Japan) while exercise with Pepper robot. The coefficient of variation of R-R intervals and high-frequency (HF) power on electrocardiograph served as indices of parasympathetic nervous activity, while the low-frequency (LF)/HF ratio served as an index of sympathetic nervous activity. Heart Rate (HR), Movement (acceleration strengths), NU (\%) shows the sympathetic-parasympathetic balance. These data are analysis results every 2 seconds.

The left side of the NU (\%) is the beginning of the exercise with Pepper robot. At the end of exercise, sympathetic nerve activity ratio has been increased.

Figure 3. The sympathetic-parasympathetic balance while exercising with Pepper robot 

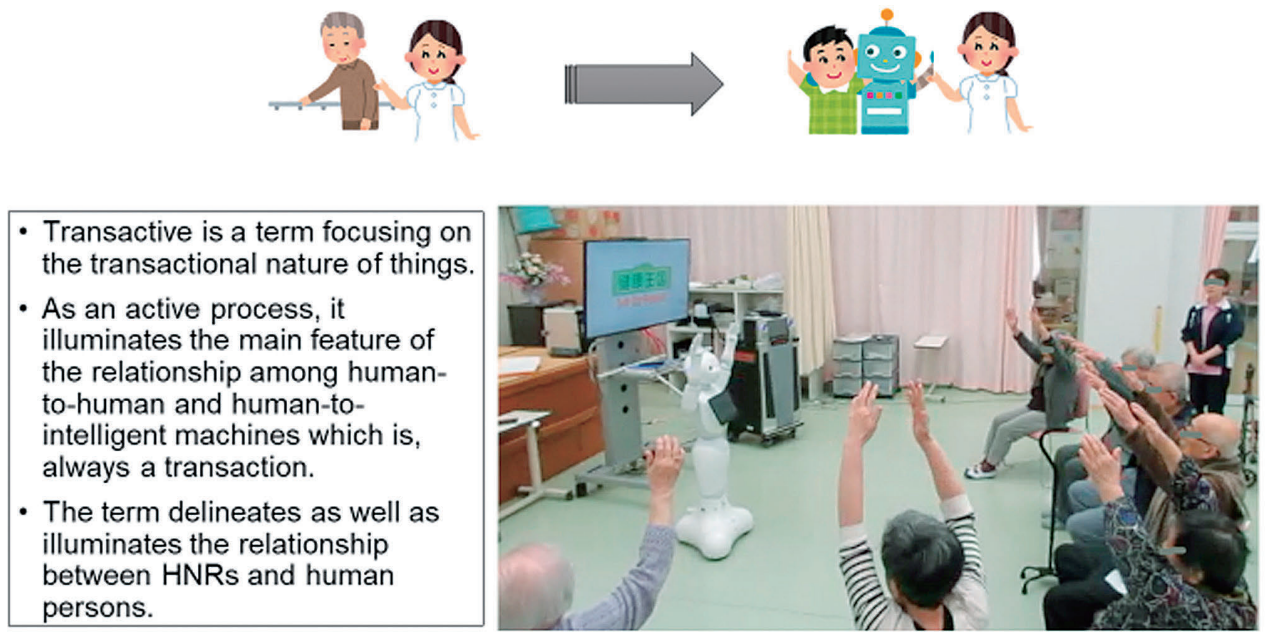

Figure 4. Nursing and rehabilitative care engagements between HNRs and human persons

CPGE. Elderly people with good physical and cognitive function can do their upper limbs exercises by Pepper's instruction, it might be promoting their quality of life.

\section{CONCLUSION}

Past research influencing human-to-human and human-to-robot relationships involving robots with artificial super-intelligence were explained and discussed to aid in possibilities of adopting appropriate programs with intelligent machines to meet the demands of future endeavors in health care. Cooperative undertakings with various interdisciplinary activities among human nurses and humanoid nursing robots will be a prospective visioning as seen and preferably realized from the perspective of Japanese human caring ideas for an aging society.

\section{CONFLICT OF INTEREST}

There is no actual or potential conflict of interest that exist in this manuscript.

\section{ACKNOWLEDGEMENTS}

This work was supported in part by JSPS KAKENHI Grant Number JP17H01609.

\section{REFERENCES}

1. Muramatsu N, Akiyama H : Japan : Super-Aging Society Preparing for the Future. The Gerontologist 51(4) : 425-432,2011

2. Annear MJ, Otani J, Sun J : Experiences of Japanese aged care : the pursuit of optimal health and cultural engagement. Age and Ageing 45(6) : 753-756, 2016

3. Shibata T, Wada K : Robot therapy : a new approach for mental healthcare of the elderly - a mini-review. Gerontology 57(4) : 378-86,2011

4. Yasuhara Y, Tamayama C, Kikukawa K, Osaka K, Tanioka T,
Watanabe N, Chiba S, Miyoshi M, Locsin R, Ren F, Fuji S, Ogasawara H, Mifune K: Required Function of the Caring Robot with Dialogue Ability for Patients with Dementia. AIA International Advanced Information Institute 4(1) : 31-42, 2012

5. Tanioka T, Yasuhara Y, Osaka K, Ito H, Locsin R, Supervising editor : Nursing Robots : Robotic Technology and Human Caring for the Elderly. Fukuro Shuppan Publishing, Okayama, Japan, 2017

6. Locsin RC, Purnell M, Tanioka T, Osaka K: Human Rights and Humanoid Relationships in Nursing and Complexity Science. In Davidson, A., Ray, M., \& Turkell, M. Nursing, Caring, and Complexity Science. Springer Publishing, New York, 2011

7. Locsin RC, Tanioka T, Campling A : In Alan Barnard \& Rozzano C. Locsin, (eds), Robots and Nursing Systems : Concepts, Relationships, and Practice. Technology and Nursing : Practice, Concepts and Issues. Palgrave-Macmillan Co., Ltd., UK, 2006

8. Sharts-Hopko NC: The coming revolution in personal care robotics : what does it mean for nurses? Nurs Adm Q 38(1) : 512,2014

9. Tanioka T, Osaka K, Locsin RC, Yasuhara Y, Ito H : Recommended Design and Direction of Development for Humanoid Nursing Robots Perspective from Nursing Researchers. Intelligent Control and Automation 8 : 96-110, 2017

10. Ito H, Miyagawa M, Kuwamura Y, Yasuhara Y, Tanioka T, Locsin RC : Professional Nurses' Attitudes towards the introduction of Humanoid Nursing Robots (HNRs) in Health Care Settings. Journal of Nursing and Health Sciences 9 : 73-81, 2015

11. Fuji S, Ito H, Yasuhara Y, Shihong H, Tanioka T, Locsin R: Discussion of Nursing Robot's Capability and Ethical Issues. INFORMATION 17(1) : 349-354, 2014

12. Tanioka $\mathrm{T}$ : The Development of the Transactive Relationship Theory of Nursing (TRETON) : A Nursing Engagement Model for Persons and Humanoid Nursing Robots. International Journal of Nursing \& Clinical Practices 4(1) : 223, 2017

13. Tanioka $\mathrm{T}$ : The Transactive Relationship Theory of Nursing. In : Tanioka T. et al., Eds., Nursing Robots : Robotic Technology and Human Caring for the Elderly. Fukuro Shuppan Publishing, Okayama, Japan, 2017

14. Fawcett $\mathrm{J}:$ On the requirements for a metaparadigm : an invitation to dialogue. Nurs Sci Q 9 : 94-97, 1996 
15. Iwasa T, Noda C, Hisashige S, Miyagawa M, Yasuhara Y, Kataoka M, Takigawa E, Edo S, Tanioka T, Ito H, Locsin RC, Mifune K: Phenomena of Transactive Relations When Using Pepper-CPGE During Occupational Therapy Activities. Proceedings of 2nd Technological Competency as Caring in the Health Sciences : 87, 2018.

16. Osaka K, Sugimoto H, Tanioka T, Yasuhara Y, Locsin R, Zhao Y, Okuda K, Saito K: Characteristics of a Transactive Phenomenon in Relationships among Older Adults with Dementia, Nurses as Intermediaries, and Communication Robot. Intelligent Control and Automation 8 : 111-125, 2017

17. White $\mathrm{CM}$ : The Nurse-Patient Encounter : Attitudes and Behaviors in Action. Journal of Gerontological Nursing 3 (3) : 16-20, 1977

18. Holopainen G, Nyström L, Kasén A : The caring encounter in nursing. Nurs Ethics Jan 1 : 969733016687161,2017

19. Snellman I, Gustafsson C, Gustafsson L : Patients' and Caregivers' Attributes in a Meaningful Care Encounter : Similarities and Notable Differences. ISRN Nursing 2012 : 320145, 2012

20. Locsin RC: The Co-Existence of Technology and Caring in the Theory of Technological Competency as Caring in Nursing. J Med Invest 64 (1.2) : 160-164, 2017

21. Locsin R: Technological Competency as Caring in Nursing :
A Model for Practice. Sigma Theta Tau International Press, Indianapolis, Indiana, 2005

22. Tanioka R, Tanioka T, Locsin R, Yasuhara Y : Potential Legal Issues and Care Implications During Care-Prevention Gymnastic Exercises for the Elderly Using Pepper in Long Term Health Care Facilities. Intelligent Control and Automation 9 (3) : 85-93, 2018

23. Vandemeulebroucke T, Dierckx BC, Gastmans C : The use of care robots in aged care : A systematic review of argumentbased ethics literature. Archives of Gerontology and Geriatrics $74: 15-25,2018$

24. Wachsmuth I : Robots Like Me : Challenges and Ethical Issues in Aged Care. Frontiers in Psychology $9:$ 432, 2018

25. Robotstart inc. : Pepper is introduced to 488 stores in all Hama Sushi! https://robotstart.info/2018/02/06/hamazushi-pepper. $\mathrm{html}$ (in Japanese)

26. Abdi J, Al-Hindawi A, Ng T, Vizcaychipi MP : Scoping review on the use of socially assistive robot technology in elderly care. BMJ Open 8(2) : e018815, 2018

27. Fuji S, Tanioka T, Yasuhara Y, Satoh M, Saito K, Purnell JM, Locsin RC, Yasui T: Characteristic Autonomic Nervous Activity of Institutionalized Elders with Dementia. Open Journal of Psychiatry 6(1) : 34-49, 2015 